\title{
Improved Mainlobe Interference Suppression Based on Blocking Matrix Preprocess
}

\author{
Jie Yang ${ }^{1}$ and Congfeng Liu $^{2}$ \\ ${ }^{1}$ School of Communication and Information, Aian University of Post \& Telecommunication, Xi'an, Shaanxi 710121, China \\ ${ }^{2}$ Research Institute of Electronic Countermeasure, Xidian University, Xian, Shaanxi 710071, China \\ Correspondence should be addressed to Congfeng Liu; cfliu@mail.xidian.edu.cn
}

Received 10 February 2015; Revised 19 March 2015; Accepted 20 March 2015

Academic Editor: John N. Sahalos

Copyright (c) 2015 J. Yang and C. Liu. This is an open access article distributed under the Creative Commons Attribution License, which permits unrestricted use, distribution, and reproduction in any medium, provided the original work is properly cited.

\begin{abstract}
For the problem of mainlobe direction shifting that is caused by the mainlobe interference suppression based on blocking matrix preprocess, an effective method is proposed which is based on the combination of diagonal loading and linear constraints. Therein, the reason for mainlobe direction shifting is analyzed and found to be that the covariance matrix mismatch exists in the realization of the adaptive beamforming. Therefore, the diagonal loading processing is used to overcome the mismatch and correct the mainlobe direction shifting, and the linear constraints are used to make sure of the beam pattern nulling in the interference directions; then the desired performance of adaptive beamforming is obtained. Simulation results attest the correctness and effectiveness of the proposed method, and they also show that the proposed method is insensitive to the selection of diagonal loading level, which means the loading factor is easy to choose.
\end{abstract}

\section{Introduction}

The adaptive array can be used to suppress the space interference effectively, especially when the interferences are located out of the mainlobe. Array antenna can realize the interference suppression by efficient adaptive beamforming algorithm; however, when the interference is located in the mainlobe, the conventional adaptive beamforming techniques will lead to the sidelobe level rising and mainbeam direction shifting; then the desired signal will be not in the beam pointing direction, and the output signal to interference plus noise ratio (SINR) will decline and the false-alarm probability will rise sharply; meanwhile the mainlobe shifting will affect the accuracy of angle estimation [1-6]. When the adaptive beamformer suppresses the mainlobe interference, it must form the null in the interference direction which is inside the mainlobe.

At present, many methods are put forward to suppress the mainlobe interference [5-9], but when the mainlobe interference is eliminated, there will be some other problems at the same time, such as the mainlobe direction shifting and sidelobe rising. Mainlobe interference suppression algorithm which is based on the blocking matrix preprocess attracted extensive attention due to the clear ideas and remarkable effect [6], but this method also leads to the problem of the mainlobe direction shifting. Therefore, this paper puts forward the new method depending on the combination of diagonal loading and linear constraints to improve the performance of the adaptive beamformer after the blocking matrix preprocess. This paper not only analyses the reason of the direction shifting of beamforming algorithm based on the blocking matrix preprocess in theory, but also gives the corresponding solution which is simple and effective in realization.

\section{Mainlobe Interference Suppression Based on Blocking Matrix Preprocess}

Assuming an interference is inside the mainlobe, in order to suppress it, we should first estimate its location in the mainlobe with the spatial spectrum estimation algorithm. Because the power of the interference is usually much stronger than that of the signal and noise, the MUSIC or other algorithms can be used, such as ESPRIT and maximum likelihood estimator $[10,11]$. The direction of arrival (DOA) of 
the mainlobe interference can be estimated based on MUSIC algorithm; the formula is given as [11]

$$
P_{\text {MUSIC }}=\frac{1}{\mathbf{a}^{H}(\theta) \mathbf{U}_{N} \mathbf{U}_{N}^{H} \mathbf{a}(\theta)},
$$

where $\mathbf{U}_{N}$ is the eigenvector matrix of the noise subspace based on the eigendecomposition of the covariance matrix $\mathbf{R}$ of the received array data. $\mathbf{a}(\theta)$ is the steering vector. The DOAs which correspond to the MUSIC spectral peak are the estimated incident directions of the signal and interference. Since the DOA of mainlobe interference is only estimated, the angle searching range only needs to be constrained in the mainlobe width, which can greatly reduce the amount of computation.

When the DOA of the mainlobe interference is obtained, take the preprocess of the mainlobe interference cancellation to the received array signals $\mathbf{X}$. Assuming the processed signals as $\mathbf{Y}$ and that there is

$$
\mathbf{Y}=\mathbf{B X}
$$

where $\mathbf{B}$ is the $(M-1) \times M$ preprocess blocking matrix, $M$ is the number of array elements, and $\mathbf{B}$ is given as

$$
\mathbf{B}=\left[\begin{array}{cccccc}
1 & -e^{-j u_{1}} & 0 & \cdots & 0 & 0 \\
0 & 1 & -e^{-j u_{1}} & \cdots & 0 & 0 \\
\vdots & \vdots & \vdots & \vdots & \vdots & \vdots \\
0 & 0 & \cdots & 1 & -e^{-j u_{1}} & 0 \\
0 & 0 & \cdots & 0 & 1 & -e^{-j u_{1}}
\end{array}\right] .
$$

In the above formula, $u_{1}=2 \pi\left(d / \lambda_{1}\right) \sin \theta_{1}, \theta_{1}$ is the azimuth of mainlobe interference, $\lambda_{1}$ is the wavelength of the narrowband interference, and $d$ is the spacing between two adjacent array elements. Actually, $\mathbf{B}$ is the blocking matrix which is used to suppress the mainlobe interference based on the adjacent antenna cancellation. Obviously, $\mathbf{Y}$ is the $M-1$ vector. Supposing the array antenna is the uniform linear array with the element spacing $d$, the received signal of the $k$ th antenna before preprocess can be expressed as

$$
x_{k}(t)=\sum_{i=0}^{P} s_{i}(t) e^{j(k-1) u_{i}}+n_{k}(t)
$$

where $k=1,2, \ldots, M ; u_{i}=2 \pi\left(d / \lambda_{i}\right) \sin \theta_{i}$ stands for the phase difference of the $i$ th signal or interference arriving at the array element between the two adjacent antenna; here, if $i=0$, index $i$ stands for signal; if $i=1,2, \ldots, P$, it stands for interference, but $s_{i}(t)$ stands for the complex envelope of the $i$ th signal or interference; $n_{k}(t)$ stands for the received noise at the $k$ th array element. After the processing with blocking matrix preprocess, we can obtain the preprocessed signal

$$
y_{k}(t)=\sum_{i=0}^{P} \bar{s}_{i}(t) e^{j(k-1) u_{i}}+\bar{n}_{k}(t),
$$

where $k=0,1, \ldots, M-1, \bar{s}_{i}(t)=s_{i}(t)\left[1-e^{j\left(u_{i}-u_{1}\right)}\right]$, and $\bar{n}_{k}(t)=n_{k}(t)-n_{k+1}(t)$. Comparing (4) and (5), the preprocess transformation changes the complex envelope of signal, but does not change the corresponding DOA; meanwhile the complex envelope $\bar{s}_{i}(t)$ equals zero for the mainlobe interference. Therefore the blocking matrix preprocess can suppress the mainlobe interference effectively and does not affect desired signal and the other interference. For the other interference suppressing inside the sidelobe with nulling, the later adaptive beamforming can be implemented. At the same time, the preprocess transformation will lose a freedom degree of the array antenna.

In [6], the preprocess transformation is first used to process the received signal, and then the adaptive beamforming is used conventionally. The array covariance matrix after transformation is

$$
\mathbf{R}_{Y}=E\left[\mathbf{Y} \mathbf{Y}^{H}\right]=\mathbf{B} \mathbf{A} \mathbf{R}_{S} \mathbf{A}^{H} \mathbf{B}^{H}+\sigma_{n}^{2} \mathbf{B} \mathbf{B}^{H},
$$

where $\mathbf{A}$ is the steering vector matrix, $\mathbf{R}_{S}$ is the signal covariance matrix, and $\sigma_{n}^{2}$ is the array noise covariance. At last, using the classical optimal weight vector algorithm, the weight vector of the adaptive beamforming is given as

$$
\mathbf{w}_{\mathrm{opt}}=\mu \mathbf{R}_{Y}^{-1} \mathbf{a}_{q},
$$

where $\mu$ is a constant and $\mathbf{a}_{q}$ is the steering vector of the desired signal. Literature [6] mentioned that if the adaptive beamforming is implemented by the above formula directly, it will lead to the problems of the sidelobe level rising and mainlobe shifting which is caused by mainlobe interference suppression; since the mainlobe shifting is caused by the preprocess transformation matrix B, so [6] puts forward the weight coefficient compensation for the adaptive weight vector which is given as follows:

$$
\mathbf{w}_{\mathrm{opt}}=\mu \mathbf{R}_{Y}^{-1}\left(\mathbf{B B}^{H}\right) \mathbf{a}_{q} .
$$

Obviously, the nature of the weight vector compensation is that the original steering vector $\mathbf{a}_{q}$ is multiplied by $\mathbf{B B}^{H}$; that is to say, $\left(\mathbf{B B}^{H}\right) \mathbf{a}_{q}$ substitutes for the steering vector $\mathbf{a}_{q}$.

Next, we will analyze the reason why the mainlobe will shift after interference suppression with blocking matrix preprocess in theory and give the efficient solution.

\section{Improved Mainlobe Interference Suppression Method}

3.1. Mismatch Analysis for Blocking Matrix Preprocess Method. Through the above analysis of the realization of the mainlobe interference suppression based on the blocking matrix preprocess which is proposed in [6], we can know that when the received array data is multiplied by the blocking matrix, according to formula (5), the preprocess can suppress the mainlobe interference effectively, but the complex envelopes of the desired signal and other interference are changed, but their DOAs do not change. We can do the following analysis for the proposed idea with the array model after the blocking matrix preprocess. 
Take the full representation for formula (5); there is

$$
y_{k}(t)=\sum_{i=0}^{P} s_{i}(t)\left[1-e^{j\left(u_{i}-u_{1}\right)}\right] e^{j(k-1) u_{i}}+\bar{n}_{k}(t) .
$$

For the mainlobe interference, when $i=1$, since $1-$ $e^{j\left(u_{i}-u_{1}\right)}=0$, so this interference need not be considered here and it does not affect the description of the received array data with the above formula. The received array data of the desired signal and other interference after the blocking matrix preprocess can be expressed as

$$
\mathbf{Y}_{1}=\mathbf{A}_{1} \mathbf{S}_{1}+\mathbf{n}_{1}
$$

In the above formula, $\mathbf{S}_{1}$ stands for the received signal which consists of the remaining sidelobe interference and desired signal vectors, except the removed mainlobe interference, but their complex envelopes have been changed as $s_{i}(t)\left[1-e^{j\left(u_{i}-u_{1}\right)}\right] ; \mathbf{A}_{1}$ stands for the steering matrix of the desired signal and remaining interferences correspondingly, $\mathbf{n}_{1}$ is the received array noise after transformation, $\mathbf{Y}_{1}$ is the received array data after blocking matrix preprocess, and actually $\mathbf{Y}_{1}$ is the same with the above $\mathbf{Y}$; in order to simplify the analysis, the symbols are uniformed and we have

$$
\mathbf{Y}_{1}=\mathbf{Y}
$$

The DOA of desired signal does not change after preprocess, but the process loses a freedom degree of array antenna; the steering vector of the desired signal and remaining interference with preprocess lose one dimension relative to the unpreprocessed steering vector; the other parameter of the steering vector does not change. Assuming the received space signals only consist of the desired signal and remaining interferences and, now, the adaptive array processing is implemented by the front $M-1$ antennas for the received array data, then the received array signal of the corresponding array can be expressed as

$$
\mathbf{Y}_{0}=\mathbf{A}_{0} \mathbf{S}_{0}+\mathbf{n}_{0}
$$

Obviously, there are

$$
\begin{gathered}
A_{1}=A_{0}, \\
S_{1}=S_{0} \Lambda,
\end{gathered}
$$

where

$$
\Lambda=\operatorname{diag}\left\{1-e^{j\left(u_{0}-u_{1}\right)}, 1-e^{j\left(u_{2}-u_{1}\right)}, \ldots, 1-e^{j\left(u_{P}-u_{1}\right)}\right\} .
$$

So we have

$$
\begin{aligned}
\mathbf{R}_{Y} & =E\left[\mathbf{Y} \mathbf{Y}^{H}\right]=E\left[\mathbf{Y}_{1} \mathbf{Y}_{1}^{H}\right]=\mathbf{A}_{1} \mathbf{R}_{S 1} \mathbf{A}_{1}^{H}+E\left[\mathbf{n}_{1} \mathbf{n}_{1}^{H}\right] \\
& =\mathbf{A}_{0} \mathbf{R}_{S 1} \mathbf{A}_{0}^{H}+\sigma_{n}^{2} \mathbf{B B}^{H}
\end{aligned}
$$

where

$$
\mathbf{R}_{S 1}=E\left[\mathbf{S}_{1} \mathbf{S}_{1}^{H}\right]=E\left[\mathbf{S}_{0} \Lambda \Lambda^{\mathbf{H}} \mathbf{S}_{0}^{H}\right]
$$

Similarly, there is

$$
\begin{aligned}
\mathbf{R}_{Y 0} & =E\left[\mathbf{Y}_{0} \mathbf{Y}_{0}^{H}\right]=\mathbf{A}_{0} \mathbf{R}_{S 0} \mathbf{A}_{0}^{H}+E\left[\mathbf{n}_{0} \mathbf{n}_{0}^{H}\right] \\
& =\mathbf{A}_{0} \mathbf{R}_{S 0} \mathbf{A}_{0}^{H}+\sigma_{n}^{2} \mathbf{I},
\end{aligned}
$$

where $\mathbf{I}$ is the identity matrix and

$$
\mathbf{R}_{S 0}=E\left[\mathbf{S}_{0} \mathbf{S}_{0}^{H}\right] .
$$

Comparing the expressions of $\mathbf{R}_{Y}$ and $\mathbf{R}_{Y 0}$, we can know that both have certain differences whether between the covariance matrix items of the desired signal and interference or between the covariance matrix items of the noise. In the adaptive beamforming, for $\mathbf{R}_{Y}$ and $\mathbf{R}_{Y 0}$, the same steering vectors are used, but the array covariance matrixes are not the same, so the difference of array processing results will exist and be obvious.

Comparing the weight vector that is solved with the ideal covariance matrix $\mathbf{R}_{Y 0}$ with that in [6] which is solved by formula (7), we can see that the adaptive beamforming process has the covariance matrix mismatch or error. Therefore it leads to the results that the mainlobe deviates from the desired signal direction. And from the above analysis, the method of weight coefficient compensation that is proposed in [6] is also difficult to obtain the desired effect.

In order to overcome the mentioned problem that exists in the proposed method in [6], this paper proposes the improved method which uses the diagonal loading to overcome the problem of the mainlobe direction shifting. Since the diagonal loading leads to the interference nulling declining, then the linear constraints are put forward to improve the performance of other interferences suppression.

3.2. Improving Beam Pointing with Diagonal Loading. Diagonal loading is a common beamforming technology; for some undetermined problems, such as when the sample number is less than array processing freedom, the sample covariance matrix will be not inversion, but diagonal loading can solve the beamforming problem [12-14]. We all know that the diagonal loading can increase the robustness of the beamformer; since diagonal loading can provide the robustness against the mismatch of the signal DOAs or array element location and the gain or phase disturbances, it can also restrain the covariance matrix mismatch that is caused by limited samples supporting or covariance matrix estimation error. Diagonal loading also can be used as a reducing dimension technology; it can shield off the influence about the eigenvector that corresponds to the small eigenvalue and then reduces the adaptive freedom degree.

Diagonal loading is realized by making the covariance matrix added with a diagonal matrixand often is formulated as follows:

$$
\widetilde{\mathbf{R}}_{Y}=\mathbf{R}_{Y}+\sigma_{L}^{2} \mathbf{I}
$$

where $\sigma_{L}^{2}$ is used to control the amount of loading and is called the loading level. 
The core and key of the diagonal loading is to select the loading level; of course the ultimate choice of loading level is determined by the adaptive beamforming algorithm or special application. If the loading level is smaller, loading effect is similar to unloading. On the contrary, if the loading level is higher, the effect is similar to no adaptiveness (the rank is 1), and the loading process shields off all the adaptive freedom. In the adaptive array signal processing, the optimal loading level is generally selected to be higher than the average power $\sigma_{n}^{2}$ of the background noise but lower than the power of the signal and interference. The experienced method in the signal processing is to select the loading level which is $5 \sim 10 \mathrm{~dB}$ higher than the power of the background noise [13]. For some robust beamformer with norm or uncertainty set constraint, the diagonal loading can be determined by the constraint parameter $[14,15]$.

Since the purpose of diagonal loading is to improve the mainlobe pointing, when the loading is very small, its improvement is not obvious; when the loading is very large, although the mainlobe can accurately point the desired direction, the interference nulling steering does not aim at the actual interference direction. These factors make the choice of loading level more difficult; from the simulation, this paper suggests that the loading level should be selected to be as large as possible; it will first ensure the accurate pointing to the desired direction and then improve the interference nulling performance by additional linear constraints. It not only meets the desired requirement for the array pattern, but also makes the choice of the loading level easier; when the selected loading level is very large, the choice and its value will be not sensitive to the beamforming algorithm. Therefore, in order to obtain the desired performance of the beam pointing, the corresponding loading level should be selected as a larger number.

3.3. Improving Other Interference Suppression with Linear Constraints. In order to obtain a desired direction signal and make other signals and noise output minimum, we can select the linear constrained minimum power (LCMP) beamformer. The weight vector of LCMP beamformer can be solved by making the output power minimum when the beamformer is under a series of linear constraints such as $\mathbf{C}^{H} \mathbf{w}=\mathbf{f}$, where $\mathbf{C}$ is the $P \times(M-1)$ constraint matrix and $\mathbf{f}$ is the $P \times 1$ constraint vector $[16,17]$.

The optimization problem of LCMP beamformer can be described as

$$
\begin{array}{ll}
\min _{\mathbf{w}} & \mathbf{w}^{H} \mathbf{R}_{Y} \mathbf{w} \\
\text { s.t. } & \mathbf{C}^{H} \mathbf{w}=\mathbf{f} .
\end{array}
$$

The optimization problem can be solved by Lagrange multiplier method. The optimal weight vector can be expressed as [17]

$$
\mathbf{w}=\mathbf{R}_{Y}^{-1} \mathbf{C}\left(\mathbf{C}^{H} \mathbf{R}_{Y}^{-1} \mathbf{C}\right)^{-1} \mathbf{f}
$$

When the diagonal loading technology is used, the corresponding weight vector can be expressed as

$$
\begin{aligned}
\widetilde{\mathbf{w}} & =\widetilde{\mathbf{R}}_{Y}^{-1} \mathbf{C}\left(\mathbf{C}^{H} \widetilde{\mathbf{R}}_{Y}^{-1} \mathbf{C}\right)^{-1} \mathbf{f} \\
& =\left(\mathbf{R}_{Y}+\sigma_{L}^{2} \mathbf{I}\right)^{-1} \mathbf{C}\left[\mathbf{C}^{H}\left(\mathbf{R}_{Y}+\sigma_{L}^{2} \mathbf{I}\right)^{-1} \mathbf{C}\right]^{-1} \mathbf{f} .
\end{aligned}
$$

Therefore, when taking the adaptive LCMP beamforming after array data preprocess, while the diagonal loading technique is also used, we can make the mainlobe point at the desired direction and form the deep nulling steering at the interference direction. In particular, the methods proposed in this paper not only have the better performance of mainlobe interference suppression, but also make the choice of the diagonal loading level very easy and simple.

\section{Simulation Analysis}

In order to validate the effectiveness of the proposed method, the simulation analysis is given as follows. By the DOA estimation and beamforming with the received array data and the preprocessed array data, the performance of the mainlobe interference suppression is verified. Through the beamforming with different processing methods, the effectiveness of the proposed method is analyzed.

Assuming the antenna is the uniform linear array, the number of array elements is $N=8$, array element spacing is half of the signal wavelength, array sampling number $K=$ 1024, signal azimuth $\theta_{0}=0^{\circ}$, there are three interferences and their azimuths are $\theta_{i}=[5,-30,40]^{\circ}$, signal to noise ratio $\mathrm{SNR}=10 \mathrm{~dB}$, and interference to noise ratio is INR = $[10,10,10] \mathrm{dB}$. Since the mainlobe width is about $20^{\circ}$, the interference with $\theta_{i}=5^{\circ}$ is inside the mainlobe.

4.1. Effectiveness Analysis. First, the MUSIC spatial spectrum estimation is used to verify the effectiveness of the mainlobe interference suppression. Then the different beamforming algorithms are taken for the original array data and the preprocess array data, and the performance of the spatial filtering for each algorithm is analyzed.

Figure 1 gives the results of MUSIC spatial spectrum estimation, wherein "MUSIC" stands for the result of MUSIC before preprocess, and "preprocess MUSIC" represents the result of MUSIC after preprocess. Obviously the blocking matrix preprocess eliminates the mainlobe interference effectively, and the preprocess array data can be used for later array processing, such as adaptive beamforming for desired signal.

Figure 2 shows the compared results of the adaptive beamforming pattern, "Capon" [18] and "preprocess" [6] stand for the Capon pattern before and after preprocess, "PP compensation" [6] represents the array pattern after preprocess and with weight vector compensation, "PP diagonal loading" [12] denotes the array pattern after preprocess and with diagonal loading, and "PP DL and linear constraint" stands for the array pattern after preprocess and with diagonal loading and linear constraints. Namely, the "Capon" beamforming uses the original array data, the other beamforming uses the array data with blocking matrix preprocess. 


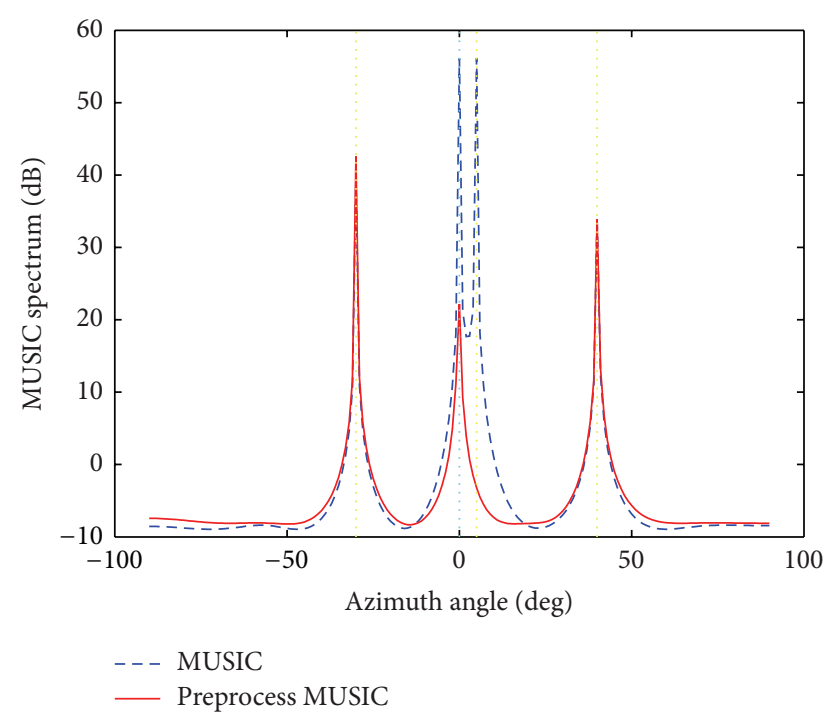

FIgURE 1: MUSIC spatial spectrum estimation (1).

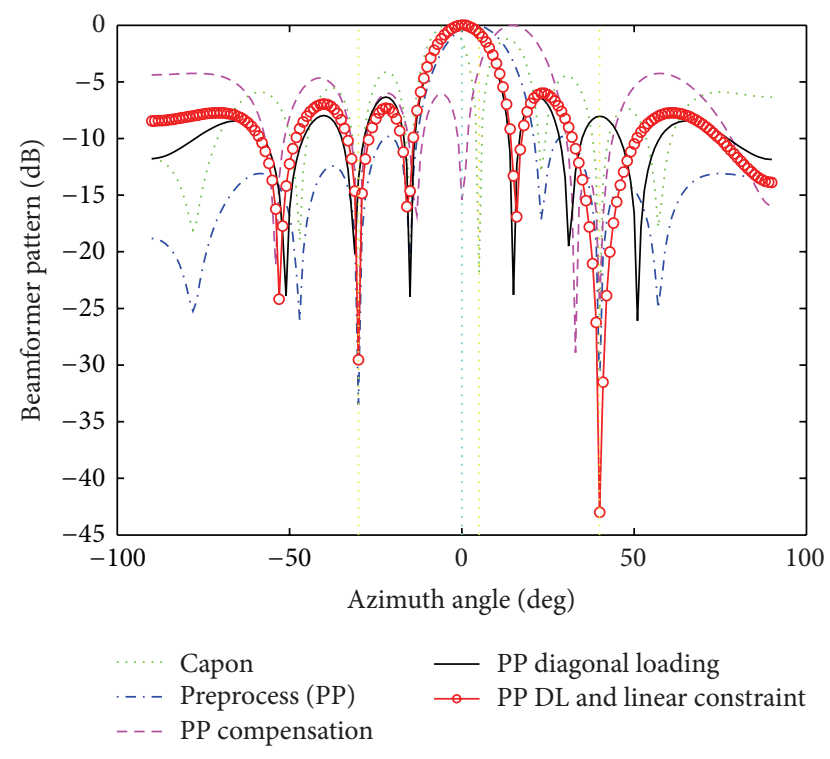

FIGURE 2: Adaptive beamforming pattern (1).

The pattern parameters, such as mainlobe direction, mainlobe width, maximum sidelobe level, and nulling depth, are given in Figure 3, wherein the abscissa is the index of the five processing methods; the index from 1 to 5 denotes the "Capon," "preprocess," "PP compensation," "PP diagonal loading," and "PP DL and linear constraint," respectively.

From the beamforming patterns in Figure 2 and comparing the pattern parameters in Figure 3, we can see that due to the interference in mainlobe the array pattern of the conventional Capon will form a deep null steering and then lead to a serious mainlobe deformation with peak shifting. The Capon pattern based on preprocess array data restrains the mainlobe interference, but the mainlobe has certain shifting; the result of weighted vector compensation is very poor. The diagonal loading after preprocess can overcome

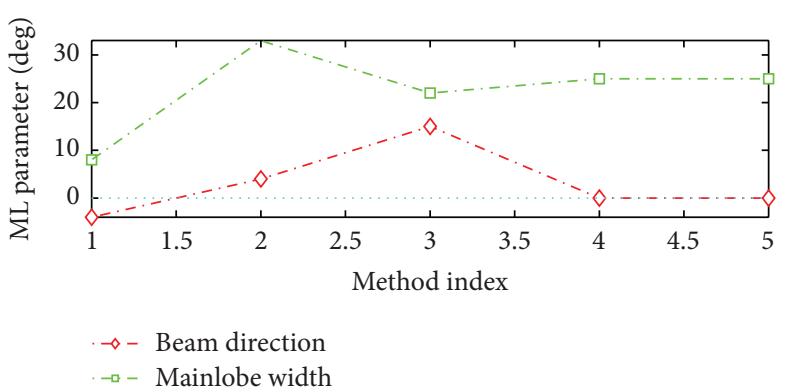

(a) Mainlobe direction and width

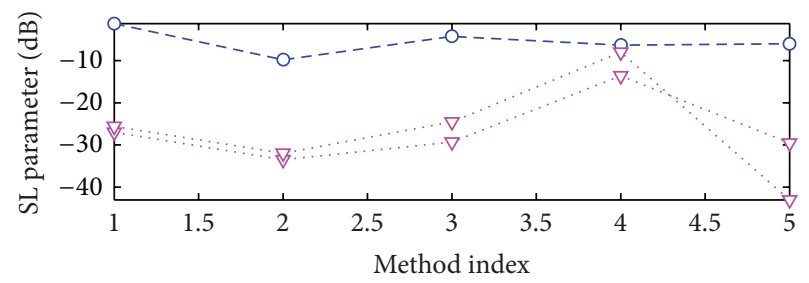

- o- Maximum sidelobe

$\because \approx$.. Nulling depth

(b) Maximum sidelobe level and nulling depth

Figure 3: Mainlobe and sidelobe parameters (1).

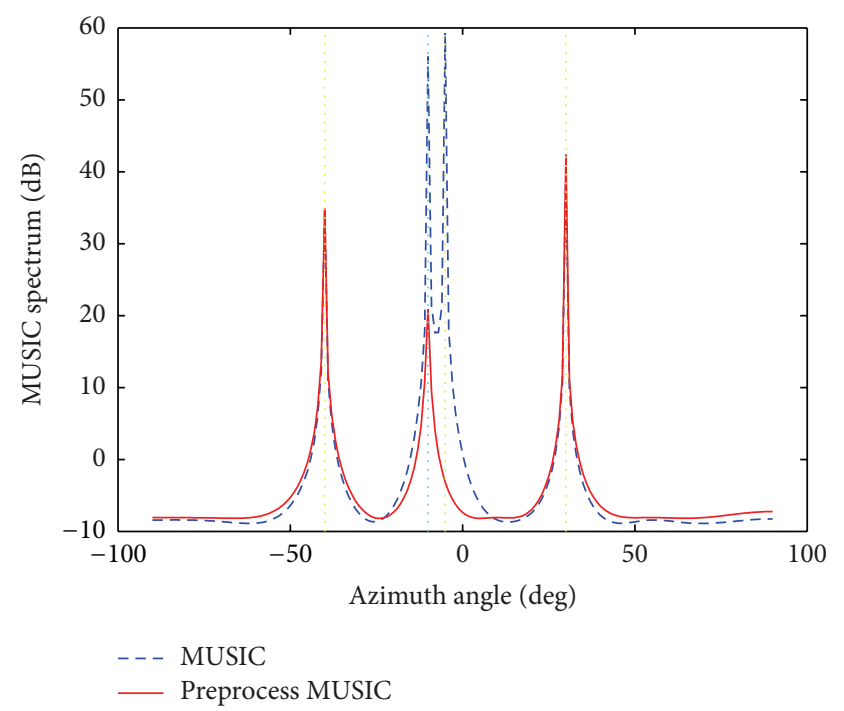

FIGURE 4: MUSIC spatial spectrum estimation (2).

the mainlobe shifting effectively, but the interference nulling is poor. The proposed method with combining the diagonal loading and linear constraints after preprocess not only realizes the mainlobe interference suppression, but also has the accurate desired pointing and interference nulling.

To further verify the correctness of the proposed algorithm, array configuration is same as former, but the azimuths of signal and interference are $\theta_{0}=-10^{\circ}$ and $\theta_{i}$ $=[-5,-40,30]^{\circ}$, respectively. The corresponding results of spatial spectrum estimation and adaptive pattern are shown in Figures 4, 5, and 6. Here, the proposed method also achieves the desired performance of the adaptive processing. 

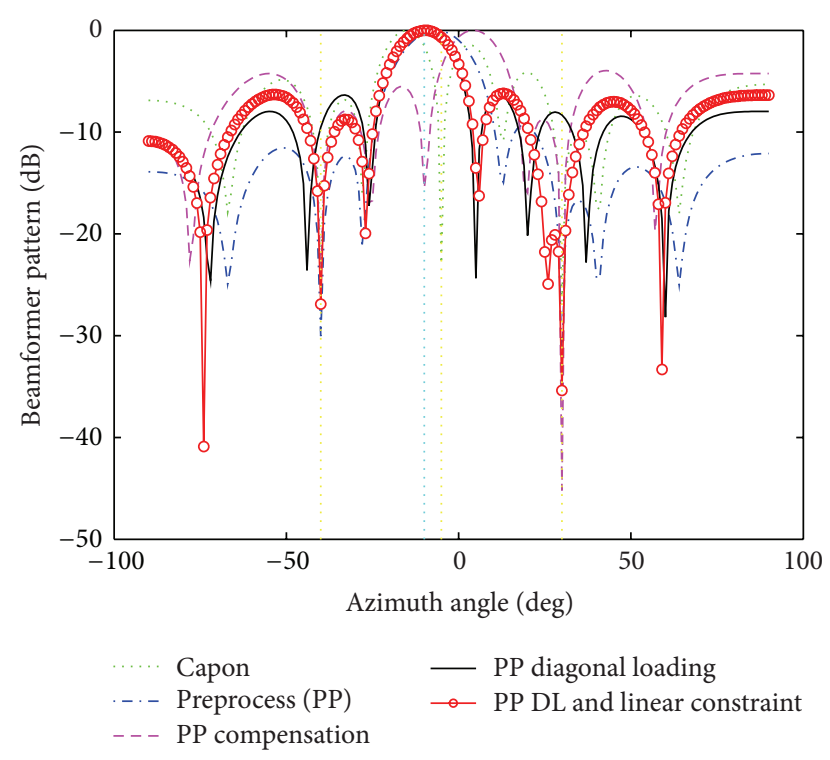

FIGURE 5: Adaptive beamforming pattern (2).

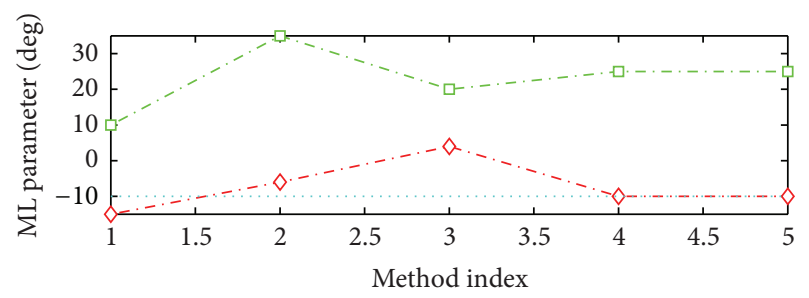

$\rightarrow-$ Beam direction

- - Mainlobe width

(a) Mainlobe direction and width

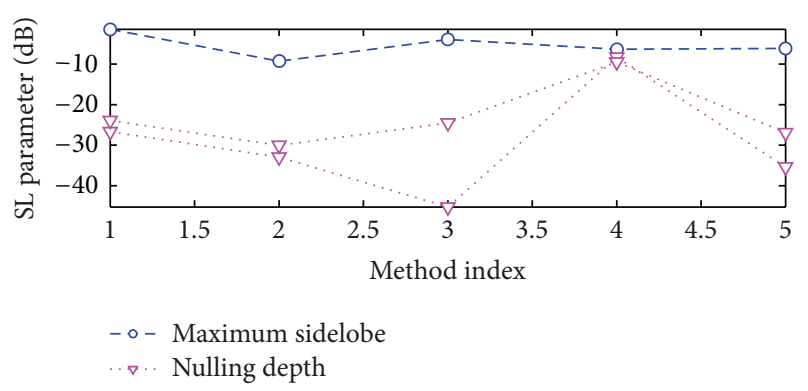

(b) Maximum sidelobe level and nulling depth

FIgURE 6: Mainlobe and sidelobe parameters (2).

Therefore, from the above comparison and analysis, we can deduce that the proposed method is correct and effective.

4.2. Diagonal Loading Analysis. In this paper, the cause of mainlobe shifting is analyzed, which is because the covariance matrix mismatch exists in adaptive beamforming. Therefore, the diagonal loading is proposed to overcome this mismatch, but how to select the loading level is the key problem in its realization. Hence, the diagonal loading level

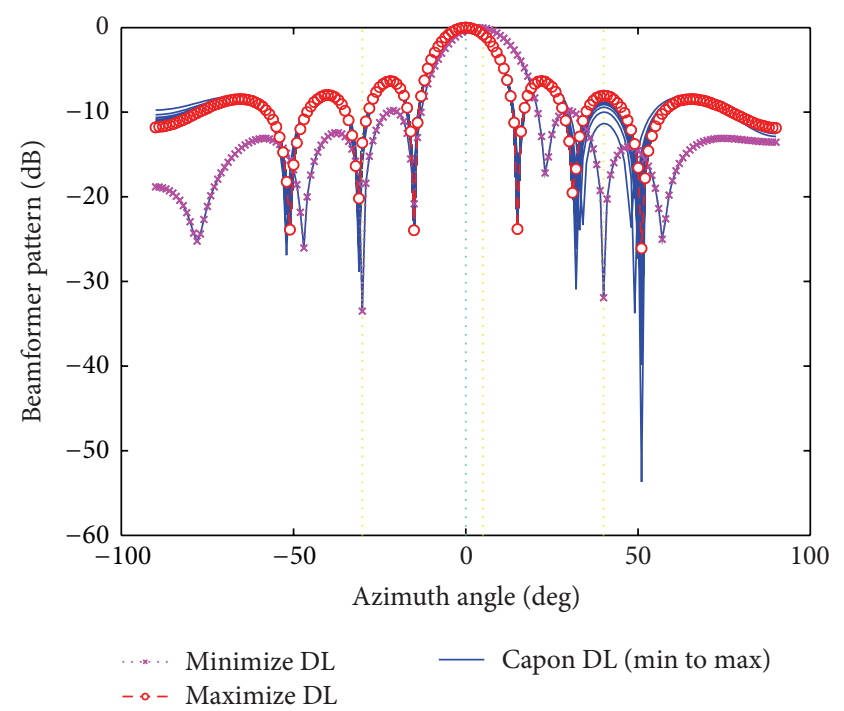

FIGURE 7: Capon pattern with diagonal loading.

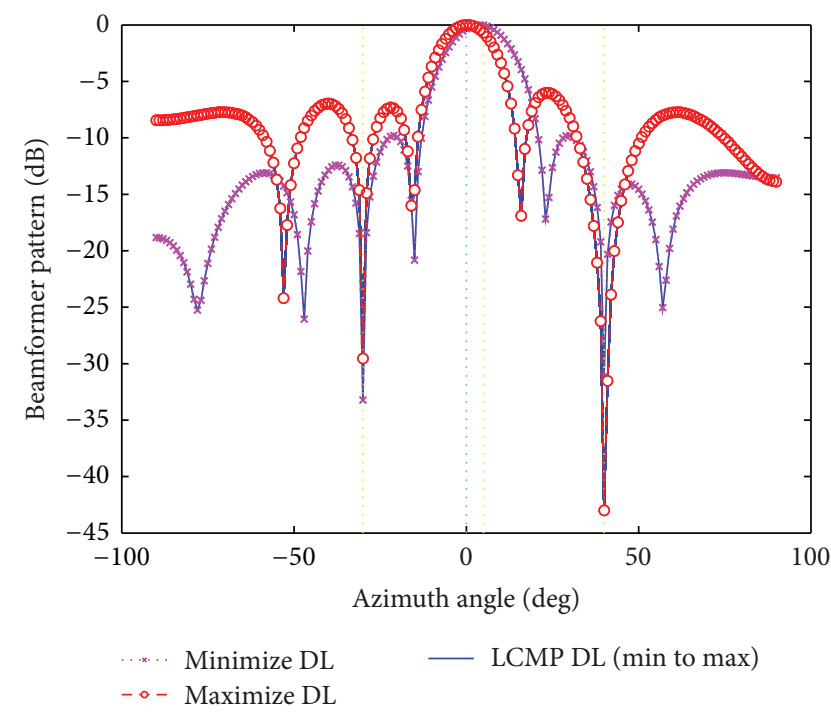

FIGURE 8: LCMP pattern with diagonal loading.

affects the performances of the traditional beamforming and the proposed method is analyzed in detail.

Figures 7 and 8 show the effects of the loading level on the beam pattern of the Capon beamforming algorithm and the LCMP beamforming algorithm after blocking matrix preprocess, respectively. Figures 9 and 10 show the pattern parameters of the mainlobe and sidelobe for Capon and LCMP beamformer with diagonal loading, respectively. Figures 11 and 12 show the output SNR curves of the corresponding adaptive beamformers, respectively.

In Figures 7 and 8, "minimize DL" and "maximize DL" stand for the patterns when the loading level takes the minimum and maximum value, respectively, wherein the minimum and maximum loading level are selected as zero and ten times the largest eigenvalue of the sample covariance matrix. "Capon DL (min to max)" and "LCMP DL 


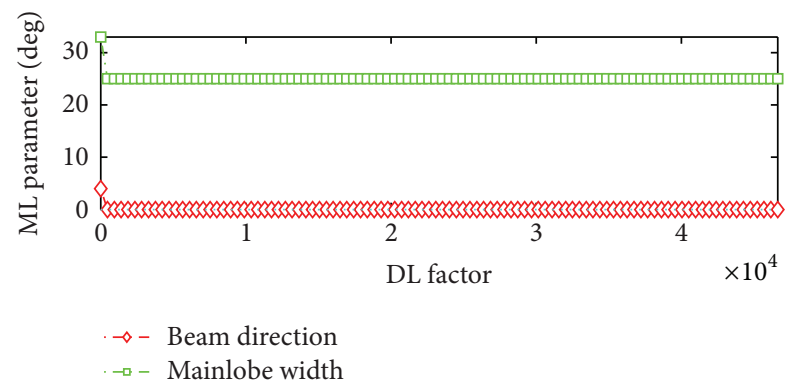

(a) Mainlobe direction and width

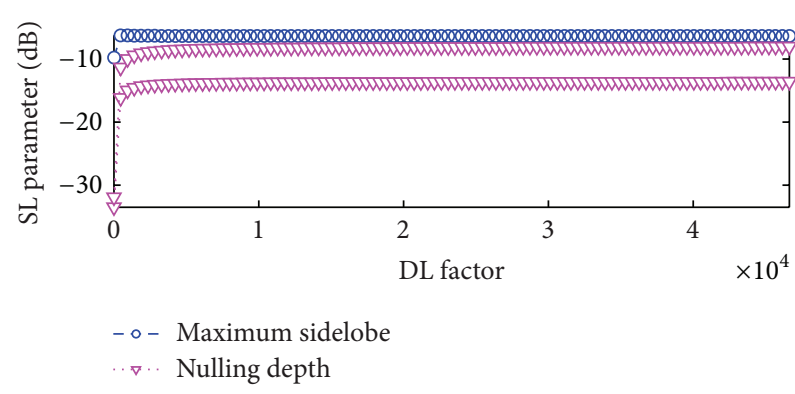

(b) Maximum sidelobe level and nulling depth

FIGURE 9: Mainlobe and sidelobe parameters of Capon beamformer with diagonal loading.

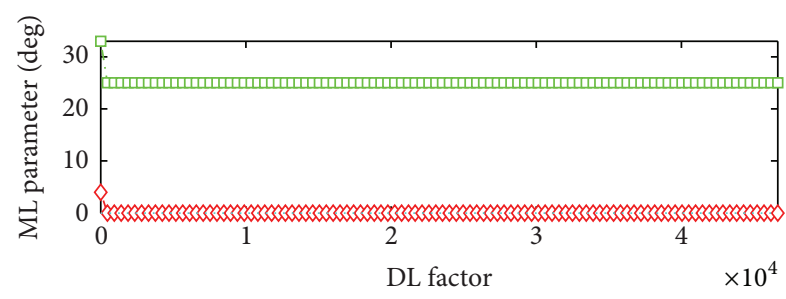

$\checkmark-$ Beam direction

$\rightarrow-$ Mainlobe width

(a) Mainlobe direction and width

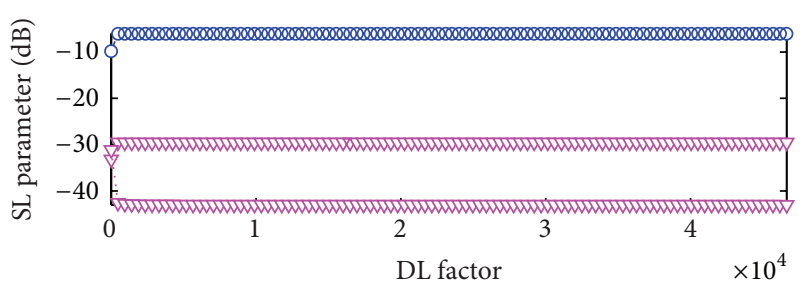

- o- Maximum sidelobe

. .. Nulling depth

(b) Maximum sidelobe level and nulling depth

FIGURE 10: Mainlobe and sidelobe parameters of LCMP beamformer with diagonal loading.

( $\min$ to $\max )$ " represent the patterns when the Capon and LCMP beamforming algorithm with the loading level take the numerical value between the minimum and maximum values, respectively.

By comparing the array patterns of the Capon and LCMP beamformers in Figures 7 and 8 and comparing their pattern

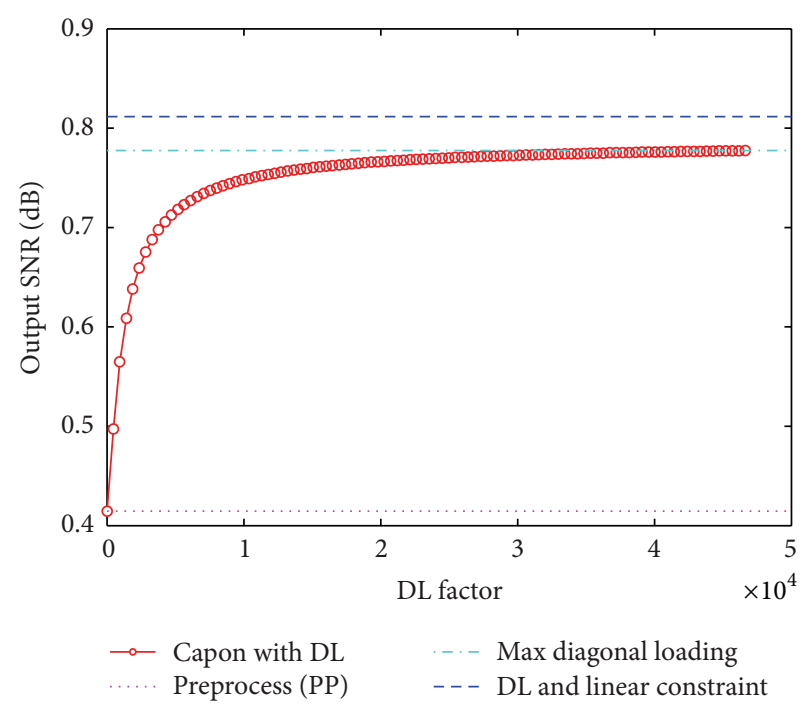

FIGURE 11: Output SNR of Capon beamformer with diagonal loading.

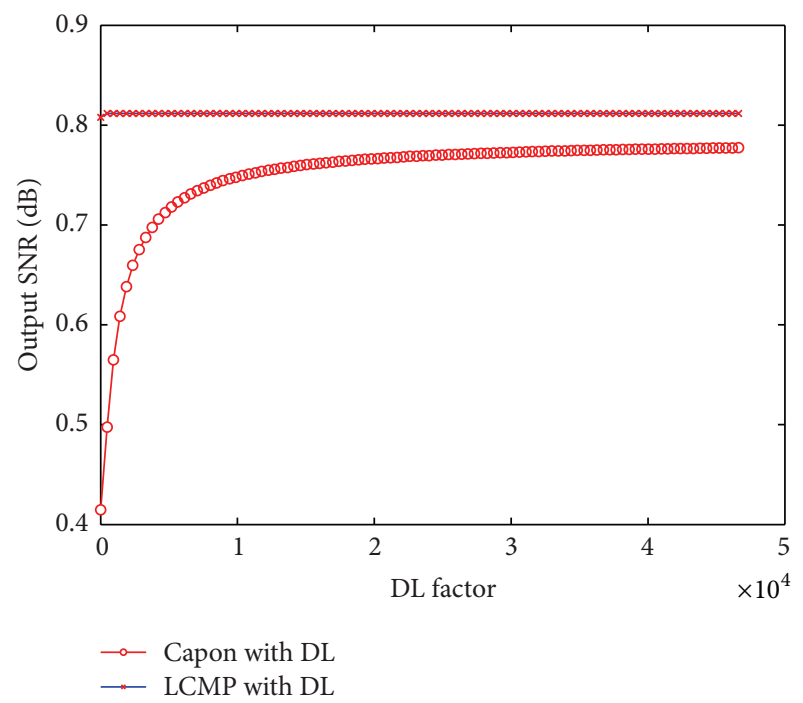

FIGURE 12: Output SNR of LCMP beamformer with diagonal loading.

parameters in Figures 9 and 10, when the loading level is selected as the minimum value, namely, zero, in fact, without diagonal loading, their mainlobes are all shifting, but when the diagonal loading is used, the two beamformers have the better mainlobe pointing performance. But the Capon beamformer has poor ability to suppress the interferences which are out of mainlobe.

In Figures 11 and 12, "Capon with DL" and "LCMP with DL" stand for the corresponding output SNRs when Capon and LCMP beamforming algorithm with diagonal loading take the numerical value between minimum and maximum values. "Preprocess" denotes the output SNR of Capon beamforming with blocking matrix preprocess; " $\max$ diagonal loading" represents the output SNR of the Capon 
beamformer with the largest diagonal loading level and blocking matrix preprocess. In Figure 11, the curve of "DL and linear constraint" is the same as that of "LCMP with DL" in Figure 12.

From Figures 11 and 12, we can see that the selection of the diagonal loading level has great effect on the Capon beamforming after blocking matrix preprocess, but, with the loading level increasing, the variation of the pattern is not very obvious; meanwhile the output SNR of the beamformer also tends to a constant. However, for the LCMP beamforming after blocking matrix preprocess, the selection of diagonal loading level has very little effect on pattern and the corresponding output SNR. Therefore, for the proposed method in this paper, the selection of loading level will be very easy and simple.

\section{Conclusion}

For the problem of mainlobe direction shifting that is caused by the mainlobe interference suppression based on blocking matrix preprocess, this paper finds that the covariance matrix error exists in the array data after the blocking matrix preprocess, which will lead to covariance matrix mismatch in the later adaptive beamforming, and then results in the mainlobe shifting. Therefore, an effective method is proposed to improve the performance of the mainlobe interference suppression which is based on the combination of diagonal loading and linear constraints, which meanwhile realizes the precise pointing of the mainlobe and other interferences nulling. Therein, the effect of diagonal loading level on the beamforming algorithms is analyzed, and it deduces that the proposed method is not sensitive to the selection of diagonal loading level; in other words, its realization is simple and effective.

\section{Conflict of Interests}

The authors declare that there is no conflict of interests regarding the publication of this paper.

\section{Acknowledgments}

The authors would like to thank the anonymous reviewers for their valuable comments. This work was supported in part by the National Natural Science Foundation (61402365, 61271300), the Shaanxi Education Natural Science Foundation (2013JK1076), the National Visiting Scholarship Program (201406965022), and the Shaanxi Industry Surmount Foundation (2013K-33, 2014KW01-04).

\section{References}

[1] A. Theil, "On combining adaptive nullsteering with high resolution angle estimation under main lobe interference conditions," IEEE Aerospace and Electronic Systems Magazine, vol. 5, no. 11, pp. 16-18, 1990.

[2] D. T. Hughes and J. G. McWhirter, "Using the penalty function method to cope with mainbeam jammers," in Proceedings of the 3rd International Conference on Signal Processing (ICSP '96), vol. 1, pp. 461-464, IEEE, Beijing, China, October 1996.

[3] K. Guney and S. Basbug, "Interference suppression of linear antenna arrays by amplitude-only control using a bacterial foraging algorithm," Progress in Electromagnetics Research, vol. 79, pp. 475-497, 2008.

[4] L. Kong and M. Luo, "Co-frequency interference suppression algorithm via maximum signal minus interference level," Progress in Electromagnetics Research, vol. 104, pp. 183-199, 2010.

[5] S. Baowei, W. Yongliang, and Z. Liangzhu, "A mainlobe interference cancelling method," in Proceedings of the International Symposium on Microwave, Antenna, Propagation and EMC Technologies for Wireless Communications (MAPE '05), pp. 2326, Beijing, China, August 2005.

[6] R. Li, Y. L. Wang, and S. H. Wan, "Research of reshaping adapted pattern under mainlobe interference conditions," Modern Radar, vol. 24, no. 3, pp. 50-55, 2002.

[7] H. Hu, H. Zhang, C.-G. Zong, and Y. Xu, "Four-channel mainlobe jamming suppression for adaptive monopulse at subarray level," Chinese Journal of Radio Science, vol. 24, no. 5, pp. 820-826, 2009.

[8] H. Han and Z. Hao, "An improved two-stage processing approach of adaptive monopulse at subarray level," Chinese Journal of Electronics, vol. 37, no. 9, pp. 1996-2004, 2009.

[9] L. Qing, Q. Huang, H.-Y. Li, and Z.-S. He, "Adaptive broadband beamforming of circular array under main lobe interference condition," Journal of the University of Electronic Science and Technology of China, vol. 38, no. 3, pp. 359-362, 2009.

[10] R. Roy and T. Kailath, "ESPRIT-estimation of signal parameters via rotational invariance techniques," IEEE Transactions on Acoustics, Speech and Signal Processing, vol. 37, no. 7, pp. 984995, 1989.

[11] R. O. Schmidt, "Multiple emitter location and signal parameter estimation," IEEE Transactions on Antennas and Propagation, vol. 34, no. 3, pp. 276-280, 1986.

[12] C. Liu and G. Liao, "Improving the STPA performance via diagonal loading," Journal of Electronics \& Information Technology, vol. 30, no. 3, pp. 906-910, 2008.

[13] B. D. Carlson, "Covariance matrix estimation errors and diagonal loading in adaptive arrays.," IEEE Transactions on Aerospace and Electronic Systems, vol. 24, no. 4, pp. 397-401, 1988.

[14] J. Li, P. Stoica, and Z. Wang, "On robust Capon beamforming and diagonal loading," IEEE Transactions on Signal Processing, vol. 51, no. 7, pp. 1702-1715, 2003.

[15] S. A. Vorobyov, A. B. Gershman, and Z.-Q. Luo, "Robust adaptive beamforming using worst-case performance optimization: a solution to the signal mismatch problem," IEEE Transactions on Signal Processing, vol. 51, no. 2, pp. 313-324, 2003.

[16] C.-F. Liu and G.-S. Liao, "Novel method of narrow band signal frequency and 2D angle estimation for wide band receiver," Chinese Journal of Electronics, vol. 37, no. 3, pp. 523-528, 2009.

[17] C. F. Liu and J. Yang, "Robust LCMP beamformer with negative loading," Progress in Electromagnetics Research, vol. 130, pp. 541561, 2012.

[18] J. Li, P. Stoica, and Z. Wang, "Doubly constrained robust Capon beamformer," IEEE Transactions on Signal Processing, vol. 52, no. 9, pp. 2407-2423, 2004. 

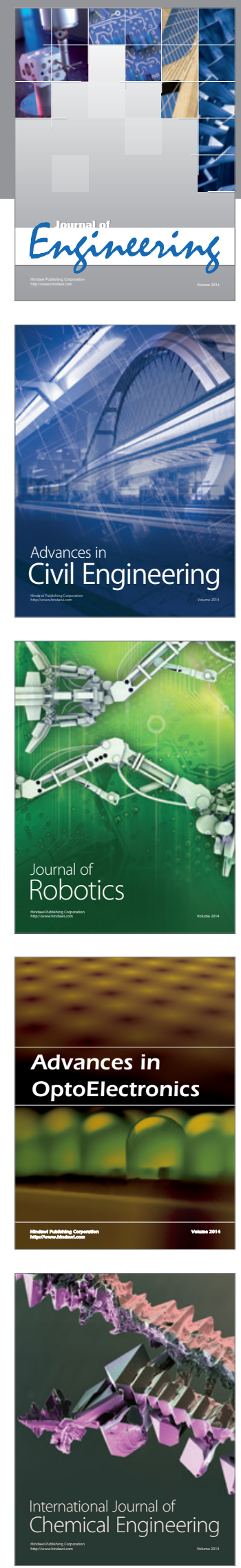

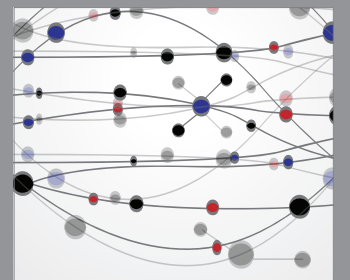

The Scientific World Journal
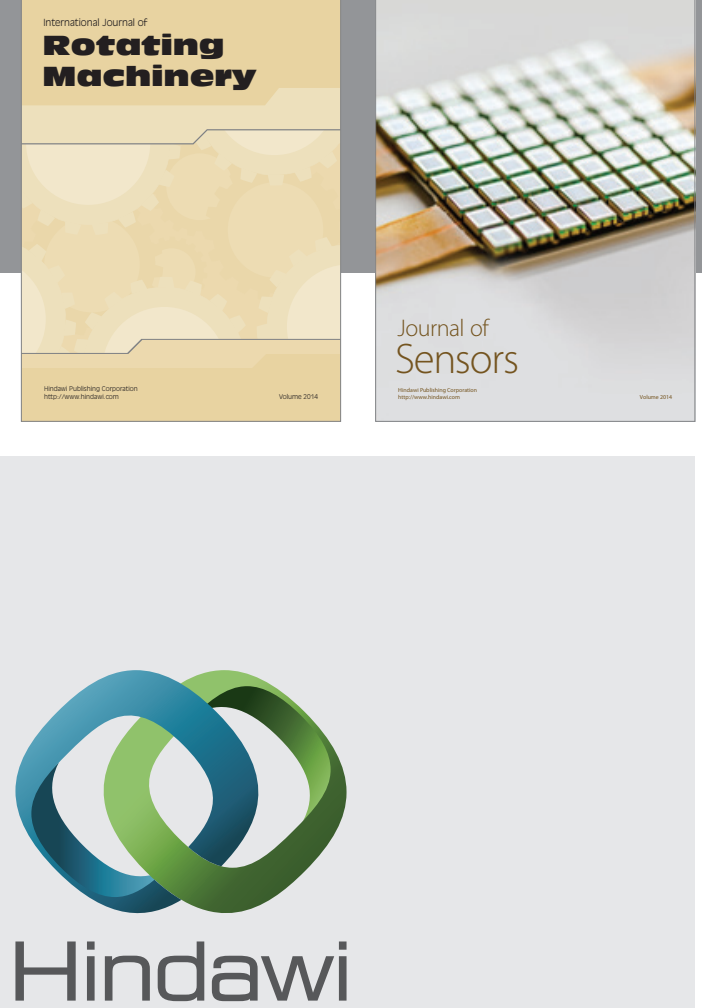

Submit your manuscripts at http://www.hindawi.com
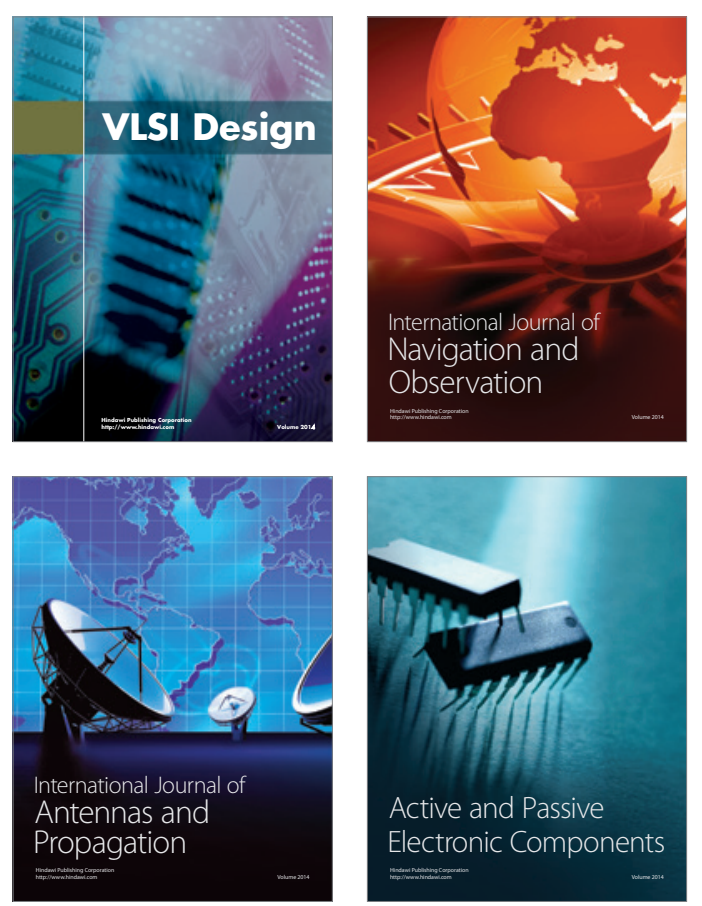
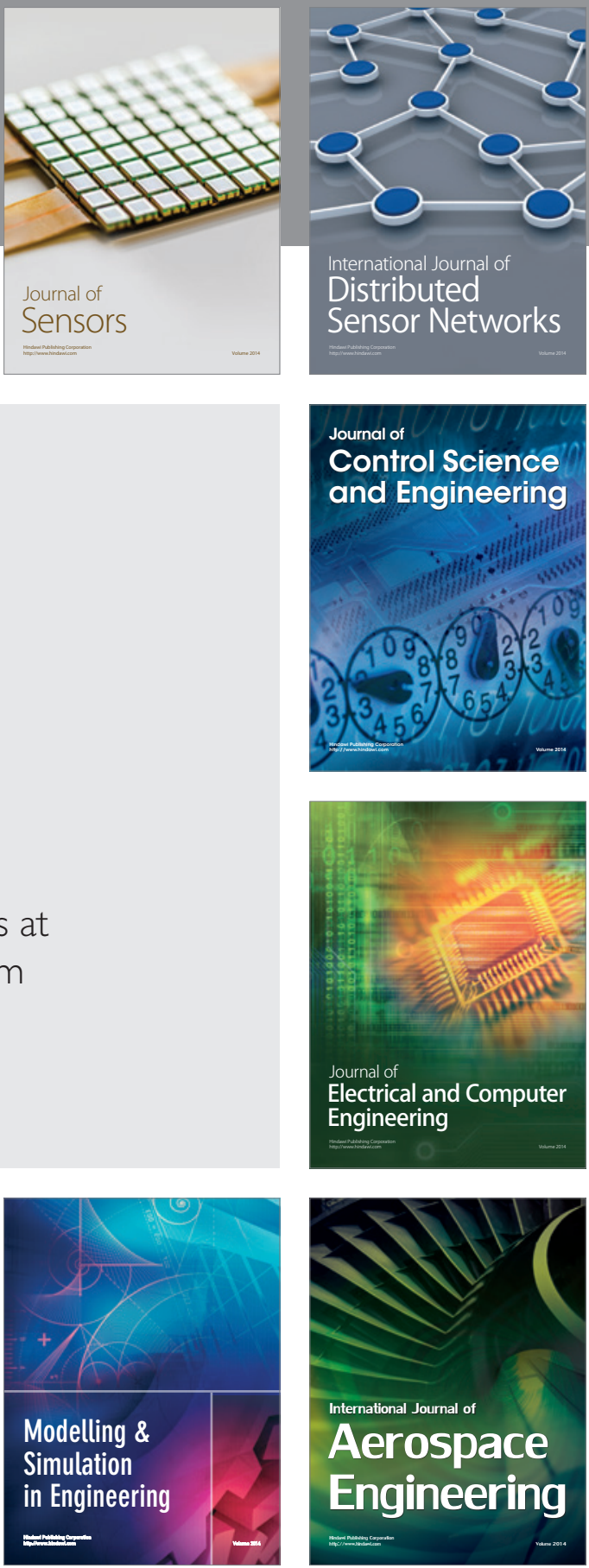

Journal of

Control Science

and Engineering
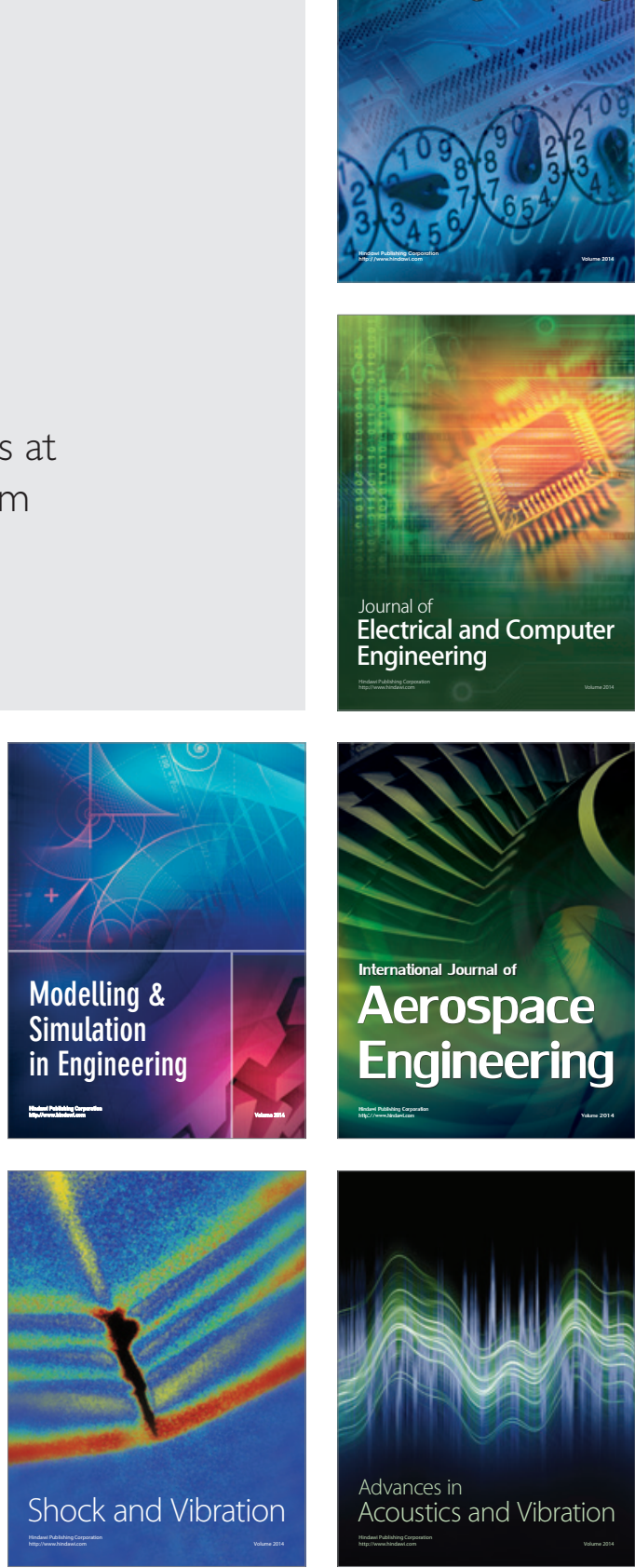